\title{
Síndrome de Cotard associada ao uso de ecstasy
}

\section{Cotard's syndrome induced by ecstasy}

Rodrigo Nicolato', Juliana Pacheco², Leandro Boson², Rodrigo Leite², João Vinícius Salgado3, Marco Aurélio Romano-Silva ${ }^{4}$, Antônio Lúcio Teixeira5 , Humberto Corrêa ${ }^{6}$

\section{RESUMO}

O termo ecstasy é usado para descrever diversas substâncias que compartilham estruturas químicas e efeitos semelhantes, referindo-se mais comumente a 3,4-metilenodioximetanfetamina (3,4-MDMA). Os efeitos psíquicos da MDMA são, sobretudo, alucinógenos e estimulantes. A tendência atual considera o delírio de Cotard como sendo a crença delirante de estar morto ou de que seus órgãos estejam paralisados ou podres, independentemente do diagnóstico do paciente. Neste artigo, relatamos o caso clínico de um paciente que

\section{Palavras-chaves}

Ecstasy, psicose, síndrome de Cotard.

\section{Key-words}

Ecstasy, psychosis, Cotard's syndrome. apresentou quadro psicótico com delírios hipocondríacos e alucinações olfativas com características de síndrome de Cotard associado ao uso crônico de ecstasy. Foi medicado com olanzapina e obteve remissão completa dos sintomas.

\section{ABSTRACT}

The term ecstasy is used to describe various substances that share similar chemical structures and effects, often referring to 3,4-methylenedioxy-N-methylamphetamine (3,4-MDMA). MDMA psychic effects are mainly hallucinatory and stimulatory. Current trends consider Cotard's delusion as a delusional belief of being dead or having paralyzed or rotten organs, independent of the diagnosis the patient has received. This case report is about a psychotic episode where the patient presented with hypochondriac delusion and olfactory hallucinations resembling Cotard's syndrome and associated with ecstasy abuse. He was given olanzapine and achieved total remission from symptoms.
1 Psiquiatra e professor de Saúde Mental da Univaço, em Ipatinga (MG). Instituto de Previdência dos Servidores de Minas Gerais. Unifenas, Belo Horizonte. Hospital de Ensino Instituto Raul Soares/FHEMIG.

2 Acadêmicos de Medicina, Univaço, Ipatinga (MG).

3 Psiquiatra. Professor de Neurociências da Universidade Fumec, em Belo Horizonte (MG). Hospital de Ensino Instituto Raul Soares/FHEMIG. 4 Psiquiatra e professor adjunto do Departamento de Farmacologia do Instituto de Ciências Biológicas da Universidade Federal de Minas Gerais (UFMG).

5 Psiquiatria, neurologista e professor adjunto do Departamento de Clínica Médica da Faculdade de Medicina da UFMG.

6 Psiquiatra, professor adjunto e chefe do Departamento de Psiquiatria, Faculdade de Medicina, UFMG. 


\section{INTRODUÇÃO}

O termo ecstasy é usado para descrever diversas substâncias que compartilham estruturas químicas e efeitos semelhantes, referindo-se mais comumente a 3,4-metilenodioximetanfetamina (3,4-MDMA). Vários estudos sobre ecstasy têm sido realizados em face de seu crescente uso, sobretudo entre jovens de classe média residentes em centros urbanos e freqüentadores de boates e festas raves. Assim, o ecstasy é usado principalmente nos finais de semana, assumindo um padrão diferente do da maioria das outras drogas, ocorrendo espaçamento médio de 2 a 3 semanas entre o consumo da droga (Cohen e Cocores, 1997).

Os efeitos psíquicos da MDMA são, sobretudo, alucinógenos e estimulantes. Segundo Lister et al. (1992), que estudaram vinte psiquiatras que usaram MDMA, os sintomas mais comuns seriam: percepção alterada do tempo (90\%), aumento da capacidade de comunicação (85\%), atenuação das defesas psicológicas (80\%), do medo (65\%), da sensação de alienação em relação aos outros (60\%), alteração da percepção visual (50\%), aumento das emoções (50\%) e diminuição da agressividade (50\%). Um discutido efeito relacionado ao uso de MDMA seria a eclosão de psicose secundária (Van Kampen e Katz, 2001).

Muito embora as descrições originais de Cotard diziam respeito a casos de melancolia ansiosa, cedo na história, o quadro descrito por Cotard foi ampliado, sendo observado em outros grupos diagnósticos. Por exemplo, em 1912, o aluno de Regis, Got, defende sua tese Contribution à l'étude du syndrome de Cotard, em que discute cerca de 30 anos de polêmica sobre o assunto. Em sua tese, Got descreve uma série de casos próprios ou de outros psiquiatras, como Capgras e Blondel. Got (1912) conclui que "a síndrome de Cotard típica, perfeitamente sistematizada, pode ser encontrada igualmente nas psicoses ansiosas agudas em indivíduos jovens". A tendência atual considera o delírio de Cotard como sendo a crença delirante de estar morto ou de que seus órgãos estejam paralisados ou podres, independentemente do diagnóstico do paciente (Berrios e Luque, 1995). Neste trabalho, relatamos o caso de uma síndrome psicótica associada ao uso periódico de ecstasy. O paciente apresentou transtorno delirante com predomínio de delírios hipocondríacos e alucinações olfativas compatíveis com a síndrome de Cotard, sendo tratado com olanzapina.

\section{RELATO DE CASO}

J. é um paciente de 23 anos, do sexo masculino, solteiro, formado em Economia, que trabalha como profissional autônomo. Não apresentava história pregressa de quadros neuropsiquiátricos nem/ou clínico-cirúrgicos, bem como antecedentes familiares de transtornos mentais. Durante a adolescência, fez uso ocasional de Cannabis sativa, sem repercussões socioocupacionais.

Há cerca de 2 anos passou a freqüentar, assiduamente, festas do tipo rave e a consumir ecstasy, em média, uma vez por semana. Referia-se à ocorrência de sintomas subjetivos, como aumento da sensibilidade a estímulos externos e da libido. Foi trazido ao serviço psiquiátrico pelos pais com a história de, há 2 meses, ter abandonado o trabalho, permanecendo isolado no quarto com higiene pessoal precária, muito angustiado, relatando que seus órgãos intestinais estavam totalmente "paralisados e putrefatos" e que não se sentia a mesma pessoa. Os pais relataram que não houve entrada de amigos na residência de J., excluindo-se, parcialmente, a possibilidade de J. usar ecstasy, durante o período em que se isolou na residência.

Ao exame inicial, o paciente apresentava-se emagrecido, desconfiado, mas sem hostilidade. Mostrava-se orientado e sem déficit cognitivo, com pontuação total de 30 pontos no Mini-Exame do Estado Mental (Bertolucci et al., 1994). A temática da putrefação e paralisação intestinal era irremovível, bem como o relato de que seus órgãos exalavam "intenso odor fétido", provocando-lhe vergonha. Não relatava sentimentos de tristeza nem quaisquer outros sintomas depressivos, como idéias de ruína, de culpa, desesperança ou morte.

Considerando tratar-se de delírio hipocondríaco e alucinação olfativa, estabeleceu-se o diagnóstico de transtorno psicótico predominantemente delirante, com sintomas de síndrome de Cotard, decorrentes do uso de estimulantes (ecstasy), mas sem associação com depressão maior. Foi orientado a manter a suspensão do ecstasy, sendo introduzida olanzapina 5 mg/dia. Realizaram-se, ainda, exames de neuroimagem (tomografia do encéfalo), eletroencefalograma e laboratoriais, incluindo hemograma completo, ionograma, glicemia, funções renal, hepática e tireoidiana, todos exames sem alterações.

Após 2 semanas do uso da olanzapina na dose de $5 \mathrm{mg} / \mathrm{dia}$, encontrava-se mais sociável, com higiene cuidada, embora ainda se referisse aos sintomas psicóticos. Optou-se por elevar a dose de olanzapina para $10 \mathrm{mg} /$ dia. Dois meses depois, o paciente retornou com remissão completa dos delírios somáticos e alucinações. Foi possível, então, reduzir progressivamente a dose da olanzapina até a suspensão em 6 meses, e o paciente retomou integralmente suas atividades laborais. No acompanhamento de 6 meses após a suspensão do medicamento, o paciente manteve-se assintomático, tendo procurado por conta própria o grupo de Narcóticos Anônimos, sem usar novamente ecstasy. 


\section{DISCUSSÃO}

No nosso conhecimento, este se trata do primeiro relato de caso na literatura de síndrome de Cotard associada ao uso de ecstasy. A história negativa, pré-mórbida e familiar, de quadros psicóticos e afetivos, o uso de ecstasy por mais de 2 anos, além de resposta completa do quadro após a suspensão da droga, corroboram a hipótese dessa associação. Convém assinalar a importância da resposta do transtorno psicótico ao uso da olanzapina.

Embora a freqüência de psicose induzida por ecstasy não seja definida, há vários relatos de casos na literatura. Sintomas psicóticos podem ocorrer durante a intoxicação aguda. Fora dessa fase, os investigadores têm enfatizado a ocorrência de psicose tanto em usuários crônicos quanto naqueles que usaram a droga em ocasiões isoladas ou esporádicas, embora os quadros psicóticos sejam mais comuns nos primeiros, sobretudo quando expostos a outras drogas (McGuire et al., 1994; Morgan, 2000). Há descrição de psicose em indivíduos geneticamente vulneráveis para transtornos psicóticos. Em contrapartida, há casos em que o paciente não possui história pessoal nem familiar de transtornos psicóticos e passa a apresentar sintomatologia psicótica prolongada, após o uso recreacional, em uma única oportunidade, de MDMA (Cohen e Cocores, 1997; Vaiva et al., 2001). De qualquer maneira, McGuire et al. (1994) defendem que não há como diferenciar a psicose funcional da induzida por ecstasy. A hipótese mais aceita sobre a gênese de fenômenos psicóticos relacionados a ecstasy considera que a depleção serotonérgica resultante da neurotoxicidade da MDMA levaria à hiperfunção dopaminérgica e, conseqüentemente, aos sintomas (Vaiva et al., 2001).

Em se tratando de transtorno psicótico induzido por drogas, a olanzapina tem sido muito eficaz e usada como primeira escolha. Em um estudo de continuação de 6 meses realizado por Landabaso et al. (2002), os sintomas psicóticos induzidos por ecstasy foram reduzidos consideravelmente com o uso de olanzapina desde o 1o mês de tratamento, com os sintomas mais significativos desaparecendo nos 3 primeiros meses, como ocorrido no caso relatado.

\section{CONCLUSÃO}

O caso clínico apresentado é compatível com transtorno psicótico predominantemente delirante (com sintomas de síndrome de (otard), induzido pelo uso de ecstasy, sem associação com depressão maior. O uso de ecstasy é preocupante, pois, além do crescente consumo da droga, pode determinar vários transtornos psiquiátricos. No diagnóstico diferencial de pacientes com sintomas psicóticos, o uso de ecstasy deve ser considerado. O quadro do paciente teve boa resposta à combinação do antipsicótico atípico olanzapina com a abstinência da droga. Assim, a olanzapina pode ser uma opção eficaz em casos de psicose secundária ao uso de ecstasy.

\section{REFERÊNCIAS}

Berrios GE, Luque R. Cotard's delusions or syndrome? Compr Psychiatr, 36: 218-23, 1995.

Bertolucci PHF, Brucki SMD, Campacci S et al. Mini-Exame do Estado Mental e Escolaridade. Arquivos de Neuropsiquiatria, 52 (1): 1-7, 1994.

Cohen RS, Cocores J. Neuropsychiatric manifestations following the use of 3,4-methylenedioxymethamphetasmine (MDMA: ecstasy). Prog Neuropsychopharmacol Biol Psychiatry, 21: 727-34, 1997.

Got J. Contribution à l'étude du Syndrome de Cotard, sa Valeur Prognostique. Bordeaux: Thése; 1912.

Landabaso MA, Iraurgi I, Jiménez-Lerma JM, Calle R, Sanz J, Gutiérrez-Fraile M. Ecstasy-induced psychotic disorder: six-month follow-up study. Eur Addict Res, 8: 133-40, 2002.

Lister M, Grob C, Bravo G, Walsh R. Phenomenology and sequelae of 3,4-methylenedioxymethamphetasmine use. J Nerv Ment Dis, 180: 345-52, 1992.

McGuire P, Cope H, Fahy T. Diversity of psychopathology associated with use of 3,4-methylenedioxymethamphetasmine ("ecstasy"). Br J Psychiatry, 165: 391-5, 1994.

Morgan MJ. Ecstasy (MDMA): a review of its possible persistent psychological effects. Psychopharmacology (Berl), 152: 230-48, 2000

Vaiva G, Bailli D, Boss V, Thomas P, Lesvastel P, Goudemand M. A case of acute psychotic episode after a single dose of ecstasy. Encephale, 27: 198-202, 2001.

Van Kampen J, Katz M. Persistent psychosis after a single ingestion of "ecstasy". Psychosomatics, 42: 525-7, 2001. 